\title{
Split $\mathrm{Ga}$ vacancies in $n$-type and semi-insulating $\boldsymbol{\beta}-\mathrm{Ga}_{2} \mathrm{O}_{3}$ single crystals
}

A. Karjalainen, ${ }^{1,2, a)}$ I. Makkonen, ${ }^{2}$ J. Etula, ${ }^{1}$ K. Goto, ${ }^{3}$ H. Murakami, ${ }^{3}$ Y. Kumagai, ${ }^{3}$ and F. Tuomisto ${ }^{1,2}$

1) Department of Applied Physics, Aalto University, P.O. Box 15100, FI-00076 Espoo,

Finland

${ }^{2)}$ Department of Physics and Helsinki Institute of Physics, University of Helsinki, P.O. Box 43, FI-00014 Helsinki,

Finland

${ }^{3}$ Department of Applied Chemistry, Tokyo University of Agriculture and Technology, 2-24-16 Naka-cho, Koganei, Tokyo, Japan

(Dated: 13 January 2021)

We report a positron annihilation study using state-of-the-art experimental and theoretical methods in $n$-type and semiinsulating $\beta-\mathrm{Ga}_{2} \mathrm{O}_{3}$. We utilize the recently discovered unusually strong Doppler broadening signal anisotropy of $\beta$ $\mathrm{Ga}_{2} \mathrm{O}_{3}$ in orientation-dependent Doppler broadening measurements, complemented by temperature-dependent positron lifetime experiments and first principles calculations of positron-electron annihilation signals. We find that split Ga vacancies dominate the positron trapping in $\beta-\mathrm{Ga}_{2} \mathrm{O}_{3}$ single crystals irrespective of type of dopant or conductivity, implying concentrations of at least $1 \times 10^{18} \mathrm{~cm}^{-3}$.

$\beta-\mathrm{Ga}_{2} \mathrm{O}_{3}$ is an emerging direct wide bandgap (4.9 eV) semiconductor with higher figures of merit for power electronics than widely used $\mathrm{GaN}$ and $\mathrm{SiC}$. As bulk single crystals with relatively low densities of extended defects can be manufactured, the role of point defects in further improvement of the $\beta-\mathrm{Ga}_{2} \mathrm{O}_{3}$ devices becomes more and more important. $\beta$ - $\mathrm{Ga}_{2} \mathrm{O}_{3}$ devices are mostly unipolar and $n$-type ${ }^{1}$ due to the lack of p-type doped material ${ }^{2}$. Cation vacancies are typical compensating acceptor defects in compound semiconductors, but very little is known about Ga vacancies in as-received $\beta-\mathrm{Ga}_{2} \mathrm{O}_{3}$ single crystals. Studies using deep level transient spectroscopy have detected four defects signals, E1, E2 and E3, E4, in as-received $\beta-\mathrm{Ga}_{2} \mathrm{O}_{3}$ crystals where all but $\mathrm{E} 4$ have been assigned to impurities ${ }^{3}$. Electron paramagnetic resonance studies on as-received $\beta-\mathrm{Ga}_{2} \mathrm{O}_{3}$ have found a shallow donor level that has also been allocated to impurities ${ }^{4}$. Photoluminescence measurements have revealed bands at $3.1 \mathrm{eV}$ and $2.9 \mathrm{eV}$, attributed to donor-acceptor pair transitions $^{5,6}$. The donor in the transition at $2.9 \mathrm{eV}$ has been suggested to be related to $V_{\mathrm{O}}$ (Refs. 6 and 7). Theoretical calculations ${ }^{3,8}$ predict that the regular (unrelaxed) Ga vacancies $\left(V_{\mathrm{Ga} 1}\right.$ and $\left.V_{\mathrm{Ga} 2}\right)$ exhibit strong relaxation to $V_{\mathrm{Ga}}^{\mathrm{ia}}, V_{\mathrm{Ga}}^{\mathrm{ib}}$ and $V_{\mathrm{Ga}}^{\text {ic }}$ split configurations, and very recently the latter have been observed and identified with scanning transmission electron microscopy ${ }^{9}$ and positron annihilation spectroscopy ${ }^{10}$.

Positron annihilation spectroscopy is a non-destructive method and particularly well adapted to studying compensating acceptor-type vacancy defects. ${ }^{11}$ Early experiments in $\beta$ $\mathrm{Ga}_{2} \mathrm{O}_{3}$ suggested $V_{\mathrm{Ga}}$ to play an important role in the electrical compensation $^{12,13}$ of $n$-type $\beta-\mathrm{Ga}_{2} \mathrm{O}_{3}$. The recently discovered unusually strong Doppler broadening signal anisotropy in $\beta-\mathrm{Ga}_{2} \mathrm{O}_{3}$ (Ref. 10) sets additional restrictions and requirements for positron experiments, but also creates a new approach for defect identification with positron annihilation without the need of defect-free reference samples. In that paper, it was shown that split Ga vacancies generate positron annihilation signals that are clearly different from regular (unrelaxed) Ga vacancies and the lattice, distinguishable by their

${ }^{a)}$ Electronic mail: antti.j.karjalainen@iki.fi particularly strong anisotropy. In this work, we utilize this strong anisotropy to detect high concentrations of split Ga vacancies in both $n$-type and semi-insulating $\beta-\mathrm{Ga}_{2} \mathrm{O}_{3}$ single crystals. We also show all crystals contain a distribution of different types of vacancy defects instead of one dominating type that is usual in, e.g., GaN and AlN. ${ }^{14,15}$ Comparing experimental and theoretically calculated positron annihilation signals allows us to conclude that split Ga vacancy - oxygen vacancy complexes are also abundant in these crystals, as suggested in Ref. 3.

The single crystals were synthesized with edge-defined film-fed growth ${ }^{16}$ (EFG) and Czochralski ${ }^{17,18}$ (CZ) methods. The EFG crystals were either unintentionally doped (uid, with free electron concentration of $5 \times 10^{17} \mathrm{~cm}^{-3}$ due to residual donors) or doped during growth with either Fe for semiinsulating behavior (resistivity $>10^{10} \Omega \mathrm{cm}$ ) or $\mathrm{Sn}$ for $n$-type behavior (free electron concentration $2 \times 10^{18} \mathrm{~cm}^{-3}$ ). The $\mathrm{CZ}$ crystals were doped with $\mathrm{Mg}$ for semi-insulating behavior (resistivity $>10^{5} \Omega \mathrm{cm}$ ). The surfaces of the plate-like samples cut from the crystals were (001) for EFG:Fe and EFG:Sn, (100) for CZ:Mg and (010) for EFG:uid. The details of the EFG and CZ bulk growth are described elsewhere. ${ }^{16-18}$

We performed positron lifetime experiments on the $\beta$ $\mathrm{Ga}_{2} \mathrm{O}_{3}$ single crystal pairs with a standard temperaturecontrolled digital spectrometer in collinear geometry and time resolution of $260 \mathrm{ps}(\mathrm{FWHM}) .1 \times 10^{6}$ counts were recorded at each temperature and the annihilations in the $1 \mathrm{MBq}^{22} \mathrm{NaCl}$ source material $(400 \mathrm{ps}, 1.5 \%), \mathrm{Al}$ foil $(210 \mathrm{ps}, 1.7 \%)$ and as positronium $(1500 \mathrm{ps}, 0.1 \%)$ were subtracted from the data before analysis. We analyzed the Doppler signal anisotropy by performing orientation-dependent experiments at room temperature with fast positrons in the same sample-source sandwich configuration as in the lifetime experiments, but mounted on a rotation stage. For further details on this setup, see Ref. 10. We note here that the energy resolution of the HPGe detector was $1.15 \mathrm{keV}$ (FWHM) at $511 \mathrm{keV}$ and $1 \times 10^{6}$ counts were accumulated in each spectrum. These experiments were complemented by performing Doppler broadening measurements in well-defined crystal orientations also with a slow positron beam, with a HPGe detector with a resolution of $1.25 \mathrm{keV}$ (FWHM) at $511 \mathrm{keV} .3 \times 10^{6}$ counts were 
accumulated at the positron implantation energy of $25 \mathrm{keV}$ where the effect of surface annihilations was insignificant. We extended our $a b$ initio theoretical calculations of the positron annihilation signals related to vacancy defects with a single missing atom (Ga vacancies and split $\mathrm{Ga}$ vacancies) presented in detail in Ref. 10 to include split Ga vacancy - oxygen vacancy complexes. The integration windows of the $S$ and $W$ parameters describing the shape of the Doppler broadened spectrum were set as $0-0.45$ a.u. $1.5-4.1$ a.u., respectively. A second $W$ parameter was defined as $2.0-4.1$ a.u. and referred to as $W 2$ throughout this work. For more detailed interpretation of the calculated Doppler spectra, we also analyze the defect-lattice ratio curves where the defect spectrum is normalized point-by-point by the lattice spectrum. Details on the experimental and modeling techniques of positron annihilation spectroscopy and associated data analysis and defect studies can be found in Ref. 11 (and the references therein), and details related to the unusual properties of $\beta-\mathrm{Ga}_{2} \mathrm{O}_{3}$ in Ref. 10.

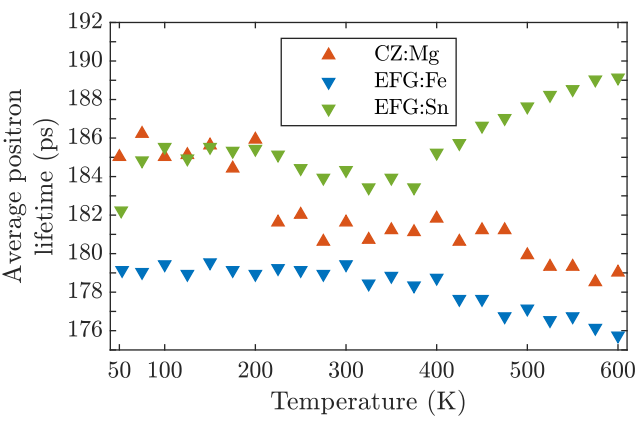

FIG. 1: The average positron lifetime measured as a function of temperature in EFG:Fe, EFG:Sn and CZ:Mg.

The average positron lifetimes measured as a function of temperature in samples $\mathrm{CZ}: \mathrm{Mg}, \mathrm{EFG}: \mathrm{Fe}$ and $\mathrm{EFG}: \mathrm{Sn}$ are shown in Fig. 1. The positron lifetime was not measured in sample EFG:uid as only one sample piece was available. Above $225 \mathrm{~K}$, the average positron lifetime behaves similarly in CZ:Mg and EFG:Fe, with the lifetime in CZ:Mg being slightly ( $3 \mathrm{ps})$ higher. The average positron lifetime of these samples decreases slowly from $225 \mathrm{~K}$ to $600 \mathrm{~K}$ by about 3 ps. On the other hand, at temperatures below $300 \mathrm{~K} \mathrm{CZ}: \mathrm{Mg}$ and EFG:Sn behave similarly, with the average lifetime increasing to $185 \mathrm{ps}$ with decreasing temperature. The average positron lifetime is rather constant at temperatures below $300 \mathrm{~K}$ in EFG:Fe, while at temperatures above $400 \mathrm{~K}$ the average positron lifetime increases with temperature in EFG:Sn. The changes with temperature are reversible. Interestingly, even if the temperature behavior of the average positron lifetime is clearly different in the three materials, at room temperature the differences are small (all data within $5 \mathrm{ps}$ ). These data show that multiple types of vacancy defects - with positron lifetimes close to each other and the lattice - are present at significant concentrations in the samples, also evident from the inability of resolving more than one component from the lifetime spectra in any of the samples or temperatures.

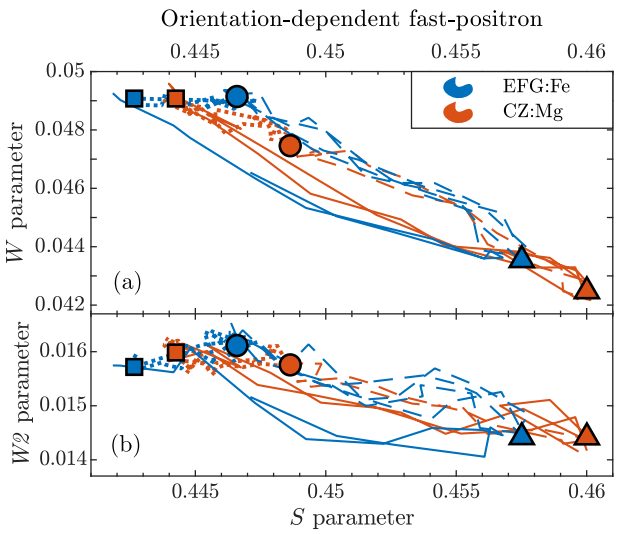

Positron beam

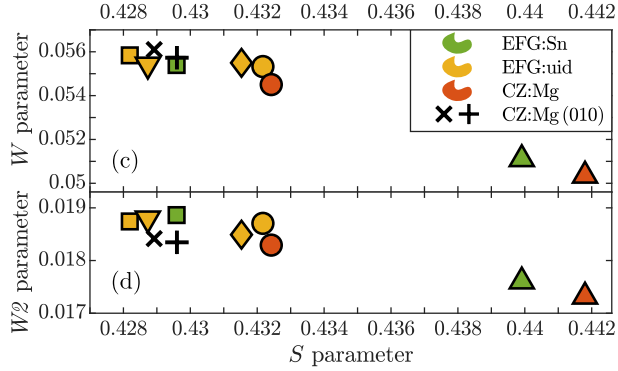

(e)

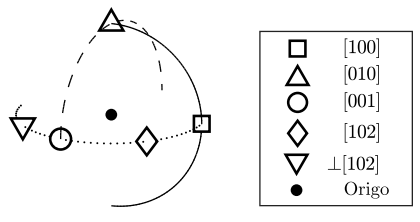

FIG. 2: (a)-(d) $S, W$ and $W 2$ parameters measured with an direction-resolved fast-positron setup and slow positron beam at room temperature. (e) The notation of the measurement directions.

The results of the orientation-dependent Doppler broadening measurements in EFG:Fe and CZ:Mg are shown in Fig. 2a)-b) and the notation is explained in Fig. 2e). CZ:Mg and EFG:Fe exhibit common trends, namely the $(S, W, W 2)$ parameters in [100] and [001] lattice directions are close to each other while [010] has equally higher $S$ and lower $W$ and $W 2$ parameters in both samples. The magnitudes of the anisotropies are also similar: 1.035 and 1.033 in $S, 0.87$ and 
0.89 in $W$, and 0.90 for both in $W 2$ parameter, respectively, in CZ:Mg and EFG:Fe. In experimental results, we define the magnitude (span) of anisotropy as the highest/lowest $S / W$ parameter divided by the lowest/highest parameter value. The main differences between CZ:Mg and EFG:Fe are that CZ:Mg has 1.003-1.006 times higher $S$ parameter, and the shape of anisotropy in CZ:Mg sample is almost linear while it is clearly loop-like in EFG:Fe. Generally, the W2 parameter (Fig. 2b)) shows same trends as the $W$ parameter, but the difference in the shape of the full anisotrop is more pronounced when the $W 2$ parameter is used in the analysis.

The slow positron beam measurements (Figs. 2c-d) deliver a similar picture of the anisotropy as the orientationdependent measurements, as seen from the data obtained in CZ:Mg with both approaches. The anisotropy spanned by samples EFG:Sn and EFG:uid is very similar to the anisotropy measured in sample EFG:Fe in the orientation-dependent experiment. CZ:Mg exhibits repeatedly similar or higher $S$ parameter compared to the EFG samples. The total anisotropy spanned by EFG:Sn and EFG:uid is 1.027 in $S, 0.92$ in $W$ and 0.94 in $W 2$ parameter, and in the case of samples CZ:Mg and $\mathrm{CZ}: \mathrm{Mg}(010)$ it is $1.033,0.90$ and 0.94 , respectively.

Importantly, the general form and span of the anisotropy indicates that trapping at split $\mathrm{Ga}$ vacancies are responsible for a significant fraction of positron annihilation signals, as discussed in Ref. 10. However, the loop-like structure in EFG:Fe, the relative differences between the $W$ and $W 2$ parameters between EFG:Sn, EFG:uid and CZ:Mg, and the non-trivial behavior of the positron lifetime as a function of temperature in all these materials strongly suggest that a more complex defect distribution has to be considered. To this end, we have calculated the positron annihilation characteristics of the following split Ga vacancy - oxygen vacancy complexes ${ }^{3}: V_{\mathrm{Ga}}^{\mathrm{ib}}-V_{\mathrm{O}}, V_{\mathrm{Ga}}^{\mathrm{ic}}-$ $V_{\mathrm{O}}, V_{\mathrm{Ga}}^{\mathrm{ib}}-2 V_{\mathrm{O}}$ and $V_{\mathrm{Ga}}^{\mathrm{ia}}-3 V_{\mathrm{O}}$

The structures of defects and the calculated positron densities are shown in Fig. 3. In $V_{\mathrm{Ga}}^{\text {ib }}-V_{\mathrm{O}}$ and $V_{\mathrm{Ga}}^{\text {ic }}-V_{\mathrm{O}}$, the positron localizes into the "half-vacancy" without $V_{\mathrm{O}}$ and, in $V_{\mathrm{Ga}}^{\mathrm{ia}}-3 V_{\mathrm{O}}$, the highest positron density locates in the half with less $V_{\mathrm{O}}$ In the symmetric $V_{\mathrm{Ga}}^{\mathrm{ib}}-2 V_{\mathrm{O}}$, the positron density is symmetric as well. The addition of $V_{\mathrm{O}}$ has only a minor effect on the calculated positron lifetimes of split Ga vacancies: ${ }^{10} V_{\mathrm{Ga}}^{\mathrm{ib}}-V_{\mathrm{O}}$ and $V_{\mathrm{Ga}}^{\mathrm{ic}}-V_{\mathrm{O}}$ have 3 ps longer lifetime than $V_{\mathrm{Ga}}^{\mathrm{ib}}$ or $V_{\mathrm{Ga}}^{\mathrm{ic}}$, while $V_{\mathrm{Ga}}^{\mathrm{ib}}-2 V_{\mathrm{O}}$ and $V_{\mathrm{Ga}}^{\mathrm{ia}}-3 V_{\mathrm{O}}$ have $5 \mathrm{ps}$ and $7 \mathrm{ps}$ longer lifetimes than $V_{\mathrm{Ga}}^{\mathrm{ib}}$ and $V_{\mathrm{Ga}}^{\mathrm{ia}}$, respectively. Defect lifetime differences of this magnitude are unlikely to be resolved in experiments.

Figure 4 shows the calculated $S, W$ and $W 2$ parameters normalised to the respective parameters of $\beta-\mathrm{Ga}_{2} \mathrm{O}_{3}$ lattice in [001] direction, following the convention adopted in Ref. 10 The $(S, W, W 2)$ parameters in the $\beta-\mathrm{Ga}_{2} \mathrm{O}_{3}$ lattice and $V_{\mathrm{Ga}}^{\mathrm{ia}}$ $V_{\mathrm{Ga}}^{\mathrm{ib}}$ and $V_{\mathrm{Ga}}^{\mathrm{ic}}$ without $V_{\mathrm{O}}$ are shown for comparison. The addition of a single $V_{\mathrm{O}}$ to $V_{\mathrm{Ga}}^{\mathrm{ib}}$ or $V_{\mathrm{Ga}}^{\mathrm{ic}}$ does not change significantly the $S$ or $W$ parameters in any crystal direction nor the shape of the anisotropy, but only slightly extends the anisotropy of the defects, mostly towards smaller $S$ (larger $W$ and $W 2$ ) parameters. The increase of anisotropy due to the addition of $V_{0}$ is less than in the case of $V_{\mathrm{Ga}}^{\mathrm{ib}}-1 \mathrm{H}$ and $V_{\mathrm{Ga}}^{\mathrm{ic}}-1 \mathrm{H}$ (Ref. 10). The addition of multiple $V_{\mathrm{O}}$ affects the Doppler broadening signa-

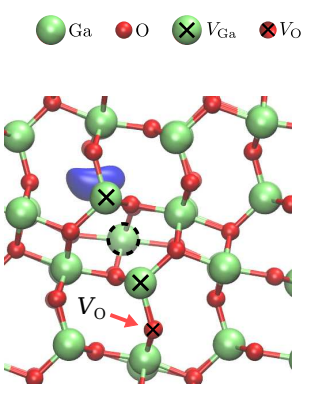

(a) $V_{\mathrm{Ga}}^{\mathrm{ib}}-V_{\mathrm{O}}$

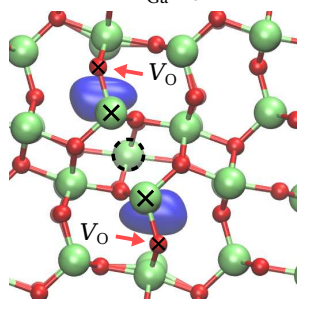

(c) $V_{\mathrm{Ga}}^{\mathrm{ib}}-2 V_{\mathrm{O}}$
$\stackrel{-\mathrm{Ga}_{\mathrm{i}}}{{ }^{[010]} \underset{[001]}{\Rightarrow}[100]}$

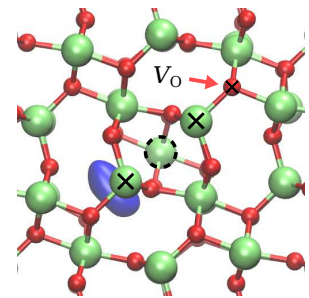

(b) $V_{\mathrm{Ga}}^{\mathrm{ic}}-V_{\mathrm{O}}$

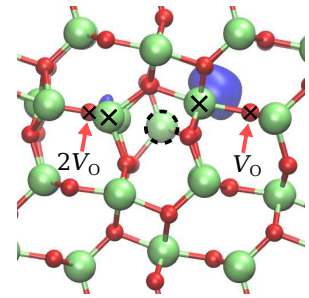

(d) $V_{\mathrm{Ga}}^{\mathrm{ia}}-3 V_{\mathrm{O}}$
FIG. 3: Calculated positron densities and the structure of the defects. The drawn positron density isosurfaces are $63 \%$ of the maximum density.

tures significantly, the anisotropies of $V_{\mathrm{Ga}}^{\mathrm{ia}}-3 V_{\mathrm{O}}$ and $V_{\mathrm{Ga}}^{\mathrm{ib}}-2 V_{\mathrm{O}}$ have the shape and span similar to $V_{\mathrm{Ga} 1}$ and $V_{\mathrm{Ga} 2}$ (Ref. 10) but with distinctly higher $S$ parameters (1.04-1.07 vs. 1.02-1.04). The vacancy-lattice ratios of the Doppler spectra (Fig. 5) show similar behavior as the $(S, W, W 2)$ parameters: $V_{\mathrm{Ga}}^{\mathrm{ib}}-V_{\mathrm{O}}$ and $V_{\mathrm{Ga}}^{\mathrm{ic}}-V_{\mathrm{O}}$ are essentially identical to $V_{\mathrm{Ga}}^{\mathrm{ib}}$ and $V_{\mathrm{Ga}}^{\mathrm{ic}}$, while the addition of $2-3 V_{\mathrm{O}}\left(V_{\mathrm{Ga}}^{\mathrm{ia}}-3 V_{\mathrm{O}}\right.$ and $\left.V_{\mathrm{Ga}}^{\mathrm{ib}}-2 V_{\mathrm{O}}\right)$ strongly enhances the $S$ parameter region and diminishes the Doppler ratio peak at 1.5 a.u. We note that except for the anisotropic features of the Doppler broadening, the addition of one or more $V_{\mathrm{O}}$ has a similar effect on the positron annihilation signals as in other compounds (e.g., GaN, InN, $\mathrm{ZnO}$ ) with significant size mismatch between cations and anions: the lifetime hardly changes for any number of $V_{\mathrm{O}}\left(\right.$ or $V_{\mathrm{N}}$ ) next to a cation vacancy, while the Doppler broadening changes only after the addition of a second $V_{\mathrm{O}}\left(\right.$ or $\left.V_{\mathrm{N}}\right){ }^{19-22}$

The combination of our experimental and theoretically calculated Doppler broadening data shows that we observe split $\mathrm{Ga}$ vacancies in all of the single crystal $\beta-\mathrm{Ga}_{2} \mathrm{O}_{3}$ samples, irrespective of type of dopant and conductivity. The contribution of these split $\mathrm{Ga}$ vacancies to the positron annihilation signals is dominant, indicating that their concentrations are at the level of $10^{18} \mathrm{~cm}^{-3}$ or higher. This is evident also in the undoped crystal (EFG:uid) that could only be measured in the lowest-anisotropy plane spanned by the [100] and [001] lattice directions (see Fig 2c). The differences in the positron lifetime 

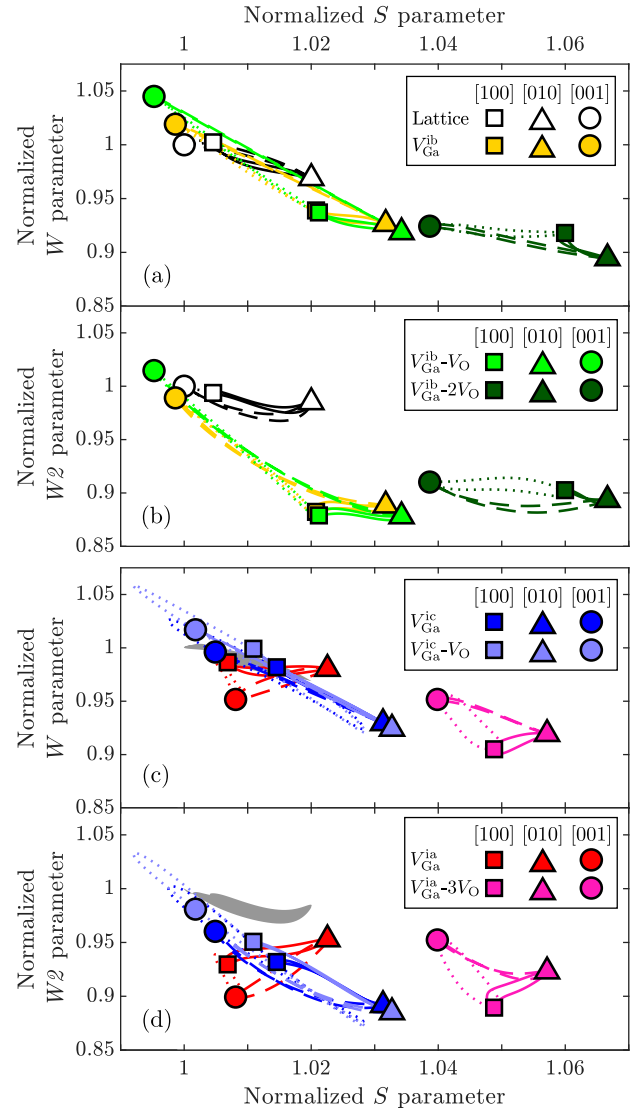

FIG. 4: Calculated $(S, W)$ and $(S, W 2)$ parameters normalised to the $\beta-\mathrm{Ga}_{2} \mathrm{O}_{3}$ lattice signal in the [001] lattice direction.

The $\beta-\mathrm{Ga}_{2} \mathrm{O}_{3}$ lattice signal is shown in (a) and (b) with white markers, and in (c) and (d) with a grey shadow. The directional notation follows that shown in Fig. 2e).

behavior as a function of temperature in samples EFG:Sn, EFG:Fe and CZ:Mg show that more than just one type of defect contributes to the data in general, and also in a single sample at least in the case of EFG:Sn and CZ:Mg.

The decreasing trend of the average lifetime above $300 \mathrm{~K}$ in the semi-insulating EFG:Fe and CZ:Mg indicates that the split $\mathrm{Ga}$ vacancies that dominate the positron annihilation are in a negative charge state, as can be expected from theoretical calculations. ${ }^{8}$ At the same time, the constant behavior of the average positron lifetime in EFG:Fe below $300 \mathrm{~K}$ suggests the data are close to saturation trapping to the split $\mathrm{Ga}$ vacancies, but as the decreasing trend at high temperatures is visible a full saturation is probably not reached. In CZ:Mg, the abrupt

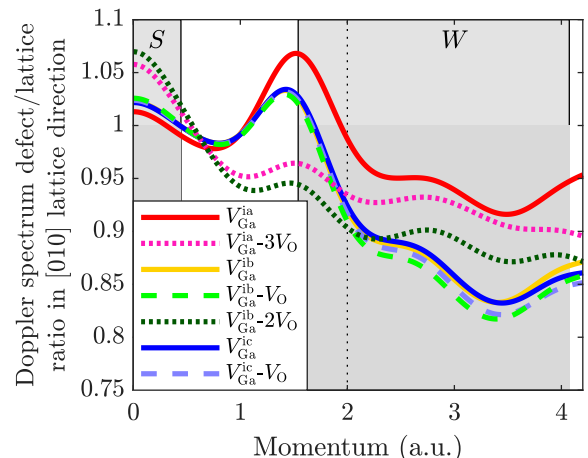

FIG. 5: Calculated defect/lattice ratio curves in the [010] lattice direction. The shaded regions show the integration windows of $(S, W)$ parameters and the dotted vertical line gives the lower bound of the $W 2$ parameter window.

jump of the average lifetime at around $200 \mathrm{~K}$ indicates that another type of defect is also present, with a charge state that changes (towards more negative when temperature is lowered) at low temperature. As the lifetime changes only a little, it is highly likely that this other type of defect is also a split $\mathrm{Ga}$ vacancy. The average lifetime is slightly but systematically higher at high temperatures in CZ:Mg than in EFG:Fe, and also the $S$ parameter is systematically higher in all measurement directions. At the same time, the $W$ parameter is systematically lower and the $W 2$ parameter similar in CZ:Mg than in EFG:Fe. This strongly suggests that in CZ:Mg the split $\mathrm{Ga}$ vacancies are complexed with one or more $V_{\mathrm{O}}$ based on comparison to calculated for $V_{\mathrm{Ga}}^{\mathrm{ib}} V_{\mathrm{Ga}}^{\mathrm{ib}}-V_{\mathrm{O}}$ and $V_{\mathrm{Ga}}^{\mathrm{ib}}-2 V_{\mathrm{O}}$. On the other hand, the loop-like anisotropy in EFG:Fe suggests that these data have a non-negligible contribution from the lattice signal, as the loop-like behavior is strengthened when going from the $(S, W)$ to $(S, W 2)$ plot in Fig. 2.

Interestingly, EFG:Sn appears to have the highest (split) Ga vacancy content as the average positron lifetime increases at higher temperatures. Hence there are some additional defects compared to, at least, EFG:Fe and CZ:Mg, while the below $300 \mathrm{~K}$ behavior suggests similar defect distribution to CZ:Mg. These additional defects trap positrons less efficiently at lower temperatures than split Ga vacancies in the other samples, as seen through the dominance of the latter below $300 \mathrm{~K}$. These could, in principle, be split Ga vacancy defects complexed with $\mathrm{Sn}$ as they should favor less negative charge states. ${ }^{9}$ Also the anisotropy span is slightly smaller in EFG:Sn (Fig 2) than in the other samples, indicating a somewhat different distribution of vacancy defects.

Finally, we note that the theoretical investigation of the added $\mathrm{O}$ vacancies on the positron annihilation spectra sheds some light on the unusually strong anisotropy of the vacancy signals in $V_{\mathrm{Ga}}^{\mathrm{ib}}$ and $V_{\mathrm{Ga}}^{\mathrm{ic}}$ split $\mathrm{Ga}$ vacancies. Interestingly, adding a second $V_{\mathrm{O}}$ to $V_{\mathrm{Ga}}^{\mathrm{ib}}-V_{\mathrm{O}}$ changes the nature anisotropy 
from the $V_{\mathrm{Ga}}^{\mathrm{ib}}$-like anisotropy to the one exhibited by $V_{\mathrm{Ga}}^{\mathrm{ia}}$ and the regular (unrelaxed) $\mathrm{Ga}$ vacancies $\left(V_{\mathrm{Ga}}\right.$ and $\left.V_{\mathrm{Ga} 2}\right)$. The main difference in the structure of $V_{\mathrm{Ga}}^{\mathrm{ib}}-2 V_{\mathrm{O}}$ compared to $V_{\mathrm{Ga}}^{\mathrm{ib}}$ $V_{\mathrm{O}}$ is that the oxygen atom nearest to the positron density is missing. Going through all the investigated vacancy structures in this work and in Ref. 10, there is a one-to-one correlation between an oxygen atom immediately "touching" the density of the localized positron and the particularly strong anisotropy in the split Ga vacancy defects. A closer look at the ratio curves in Ref. 10 and Fig. 5 shows that the presence of this oxygen atom increases the intensity of the Doppler broadening spectrum at 1-1.5 a.u. (peak/shoulder region) particularly in the orientations perpendicular to [010]. Adjacent $\mathrm{O}$ atoms have been observed to strongly alter the Doppler broadening characteristics also in other semiconductors, for example $\mathrm{Si}^{23}$ This highlights the importance of having a sufficiently detailed picture of the crystal structure and the atomic configurations around the vacancy for reliable defect identification.

In summary, we utilized the unusually strong Doppler signal anisotropy of $\beta-\mathrm{Ga}_{2} \mathrm{O}_{3}$ found in Ref. 10 to study vacancy defects in $n$-type and semi-insulating $\beta-\mathrm{Ga}_{2} \mathrm{O}_{3}$ single crystals. The orientation-dependent measurements on the Doppler signal anisotropy combined with temperaturedependent positron lifetime experiments and state-of-the ar first principles calculations of the positron annihilation signals reveal that all the studied $\beta-\mathrm{Ga}_{2} \mathrm{O}_{3}$ single crystals contain high concentrations $\left(>1 \times 10^{18} \mathrm{~cm}^{-3}\right)$ of split Ga vacancies, irrespective of conduction type. The details of the defect distributions are different in different crystals. The Sn-doped $n$-type crystals exhibited highest (split) Ga vacancy concentrations and the Fe-doped semi-insulating the lowest, as expected for thermodynamics-driven defect formation that is typical for melt-grown crystals.

\section{ACKNOWLEDGMENTS}

We thank Prof. Lasse Vines and Dr. Joel B. Varley for fruitful discussions, and Dr. Zbigniew Galazka for providing the $\mathrm{CZ}$ samples. We acknowledge the computational resource provided by the CSC (Finnish IT Centre for Science). A. Karjalainen wishes to thank the Magnus Ehrnrooth Foundation for financial support.

\section{AIP PUBLISHING DATA SHARING POLICY}

The data that support the findings of this study are available from the corresponding author upon reasonable request.

${ }^{1}$ M. Higashiwaki, H. Murakami, Y. Kumagai, and A. Kuramata, "Curren status of $\mathrm{Ga}_{2} \mathrm{O}_{3}$ power devices," Jpn. J. Appl. Phys. 55, $1202 \mathrm{~A} 1$ (2016).

${ }^{2}$ A. Kyrtsos, M. Matsubara, and E. Bellotti, "On the feasibility of p-type $\mathrm{Ga}_{2} \mathrm{O}_{3}, "$ Appl. Phys. Lett. 112, 032108 (2018).

${ }^{3}$ M. Ingebrigtsen, A. Y. Kuznetsov, B. Svensson, G. Alfieri, A. Mihaila,

U. Badstübner, A. Perron, L. Vines, and J. Varley, "Impact of proton irradiation on conductivity and deep level defects in $\beta-\mathrm{Ga}_{2} \mathrm{O}_{3}$," APL Mater 7, 022510 (2019)
${ }^{4}$ H. J. von Bardeleben, S. Zhou, U. Gerstmann, D. Skachkov, W. R. L. Lambrecht, Q. D. Ho, and P. Deák, "Proton irradiation induced defects in $\beta$ $\mathrm{Ga}_{2} \mathrm{O}_{3}$ : A combined EPR and theory study," APL Materials 7, 022521 (2019), https://doi.org/10.1063/1.5053158.

${ }^{5} \mathrm{~L}$. Binet and D. Gourier, "Origin of the blue luminescence of $\beta-\mathrm{Ga}_{2} \mathrm{O}_{3}$," Journal of Physics and Chemistry of Solids 59, 1241 - 1249 (1998).

${ }^{6}$ T. T. Huynh, L. L. C. Lem, A. Kuramata, M. R. Phillips, and C. Ton-That, "Kinetics of charge carrier recombination in $\beta-\mathrm{Ga}_{2} \mathrm{O}_{3}$ crystals," Phys. Rev. Materials 2, 105203 (2018).

${ }^{7}$ T. Onuma, S. Fujioka, T. Yamaguchi, M. Higashiwaki, K. Sasaki, T. Masui, and T. Honda, "Correlation between blue luminescence intensity and resistivity in $\beta-\mathrm{Ga}_{2} \mathrm{O}_{3}$ single crystals," Applied Physics Letters 103, 041910 (2013), https://doi.org/10.1063/1.4816759.

${ }^{8}$ J. B. Varley, H. Peelaers, A. Janotti, and C. G. Van de Walle, "Hydrogenated cation vacancies in semiconducting oxides," J. Phys.: Condens. Matter 23, 334212 (2011)

J. M. Johnson, Z. Chen, J. B. Varley, C. M. Jackson, E. Farzana, Z. Zhang, A. R. Arehart, H.-L. Huang, A. Genc, S. A. Ringel, C. G. Van de Walle, D. A. Muller and J. Hwang "Unusual formation of point-defect complexes in the ultrawide-band-gap semiconductor $\beta-\mathrm{Ga}_{2} \mathrm{O}_{3}$," Phys. Rev. X 9. 041027 (2019).

${ }_{10}$ A. Karjalainen, V. Prozheeva, K. Simula, I. Makkonen, V. Callewaert, J. B. Varley, and F. Tuomisto, "Split Ga vacancies and the unusually strong anisotropy of positron annihilation spectra in $\beta-\mathrm{Ga}_{2} \mathrm{O}_{3}$," Phys. Rev. B 102, 195207 (2020)

${ }^{11} \mathrm{~F}$. Tuomisto and I. Makkonen, "Defect identification in semiconductors with positron annihilation: Experiment and theory," Rev. Mod. Phys. 85, $1583(2013)$.

${ }^{12}$ E. Korhonen, F. Tuomisto, D. Gogova, G. Wagner, M. Baldini, Z. Galazka, R. Schewski, and M. Albrecht, "Electrical compensation by Ga vacancies in $\mathrm{Ga}_{2} \mathrm{O}_{3}$ thin films," Appl. Phys. Lett. 106, 242103 (2015).

${ }^{13}$ F. Tuomisto, A. Karjalainen, V. Prozheeva, I. Makkonen, G. Wagner, and M. Baldini, "Ga vacancies and electrical compensation in $\beta-\mathrm{Ga}_{2} \mathrm{O}_{3}$ thin films studied with positron annihilation spectroscopy," in Oxide-based Materials and Devices X, Vol. 10919 (International Society for Optics and Photonics, 2019) p. 1091910

${ }^{14}$ K. Saarinen, T. Laine, S. Kuisma, J. Nissilä, P. Hautojärvi, L. Dobrzynski, J. M. Baranowski, K. Pakula, R. Stepniewski, M. Wojdak, A. Wysmolek, T. Suski, M. Leszczynski, I. Grzegory, and S. Porowski, "Observation of native Ga vacancies in GaN by positron annihilation," Phys. Rev. Lett. 79, 3030 (1997).

${ }^{15}$ J.-M. Mäki, I. Makkonen, F. Tuomisto, A. Karjalainen, S. Suihkonen, J. Räisänen, T. Y. Chemekova, and Y. N. Makarov, "Identification of the $V_{\mathrm{Al}}-\mathrm{O}_{\mathrm{N}}$ defect complex in AIN single crystals," Phys. Rev. B 84, 081204 (2011)

${ }^{16}$ A. Kuramata, K. Koshi, S. Watanabe, Y. Yamaoka, T. Masui, and S. Yamakoshi, "High-quality $\beta$ - $\mathrm{Ga}_{2} \mathrm{O}_{3}$ single crystals grown by edge-defined film-fed growth," Japanese Journal of Applied Physics 55, 1202A2 (2016).

${ }^{17}$ Z. Galazka, K. Irmscher, R. Uecker, R. Bertram, M. Pietsch, A. Kwasniewski, M. Naumann, T. Schulz, R. Schewski, D. Klimm, and M. Bickermann, "On the bulk $\beta-\mathrm{Ga}_{2} \mathrm{O}_{3}$ single crystals grown by the Czochralski ermann, "On the bulk $\beta-\mathrm{Ga}_{2} \mathrm{O}_{3}$ single crystals grown by the
method," Journal of Crystal Growth 404, $184-191$ (2014).

${ }^{18}$ Z. Galazka, R. Uecker, D. Klimm, K. Irmscher, M. Naumann, M. Pietsch, A. Kwasniewski, R. Bertram, S. Ganschow, and M. Bickermann, "Scalingup of bulk $\beta-\mathrm{Ga}_{2} \mathrm{O}_{3}$ single crystals by the Czochralski method," ECS Journal of Solid State Science and Technology 6, Q3007-Q3011 (2016).

${ }^{19} \mathrm{C}$. Rauch, I. Makkonen, and F. Tuomisto, "Identifying vacancy complexes in compound semiconductors with positron annihilation spectroscopy: A case study of InN," Phys. Rev. B 84, 125201 (2011).

${ }^{20}$ A. Uedono, S. Ishibashi, S. F. Chichibu, and K. Akimoto, "Point defects in $\mathrm{GaN}$ and related group-III nitrides studied by means of positron annihilation," Proc. SPIE 7939, 79390I (2011)

${ }^{21}$ I. Makkonen, E. Korhonen, V. Prozheeva, and F. Tuomisto, "Identification of vacancy defect complexes in transparent semiconducting oxides $\mathrm{ZnO}$, $\mathrm{In}_{2} \mathrm{O}_{3}$ and $\mathrm{SnO}_{2}$," J. Phys.: Condens. Matt. 28, 224002 (2016)

${ }^{22}$ F. Tuomisto, V. Prozheeva, I. Makkonen, T. H. Myers, M. Bockowski, and H. Teisseyre, "Amphoteric Be in GaN: Experimental evidence for switching between substitutional and interstitial lattice sites," Phys. Rev. Lett. 119 196404 (2017) 
${ }^{23}$ A. C. Kruseman, A. van Veen, H. Schut, P. E. Mijnarends, and M. Fujinami, "Buried oxide and defects in oxygen implanted Si monitored by

https://doi.org/10.1063/1.1380411. positron annihilation," Journal of Applied Physics 90, 1179-1187 (2001), 


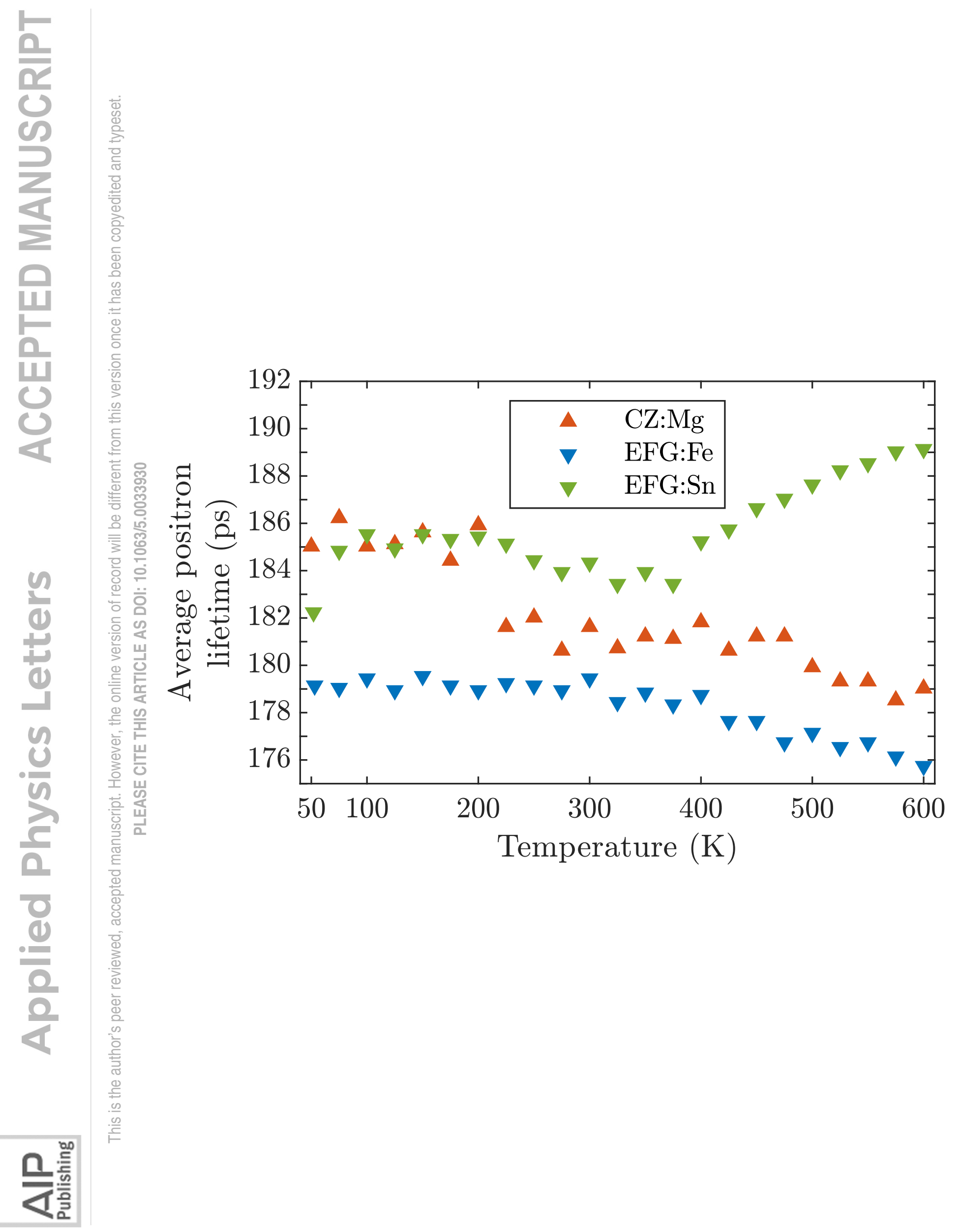


Orientation-dependent fast-positron

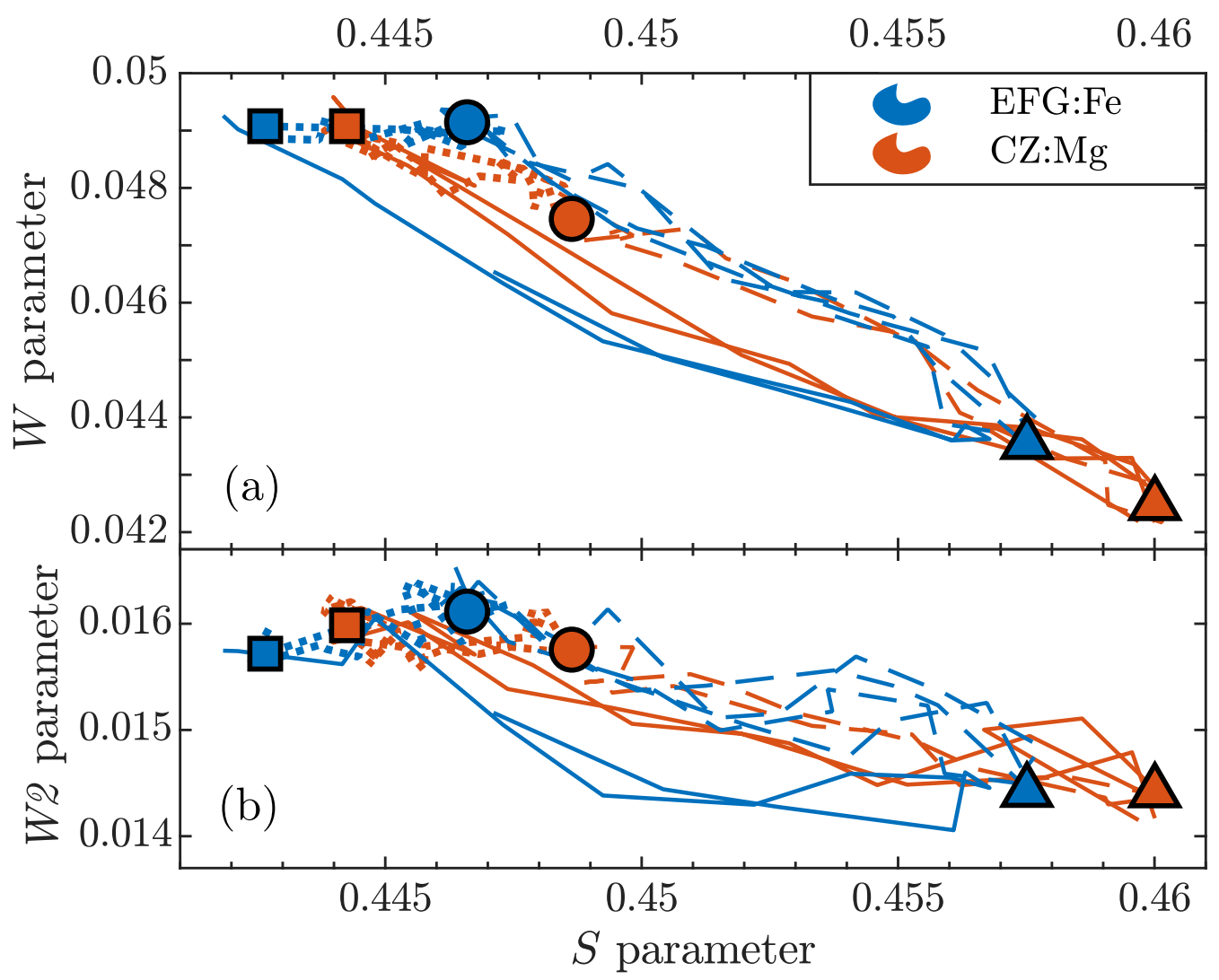

Positron beam

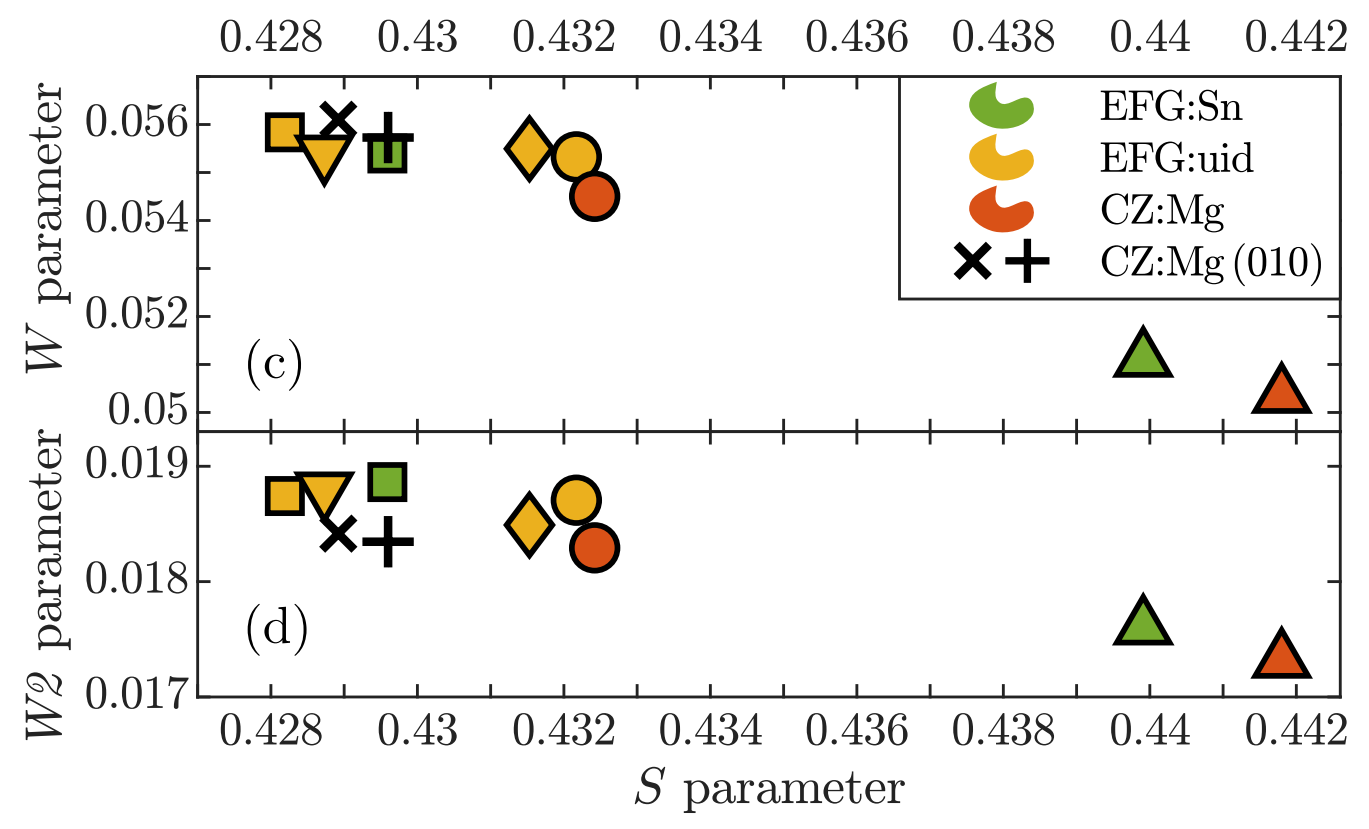


(e)

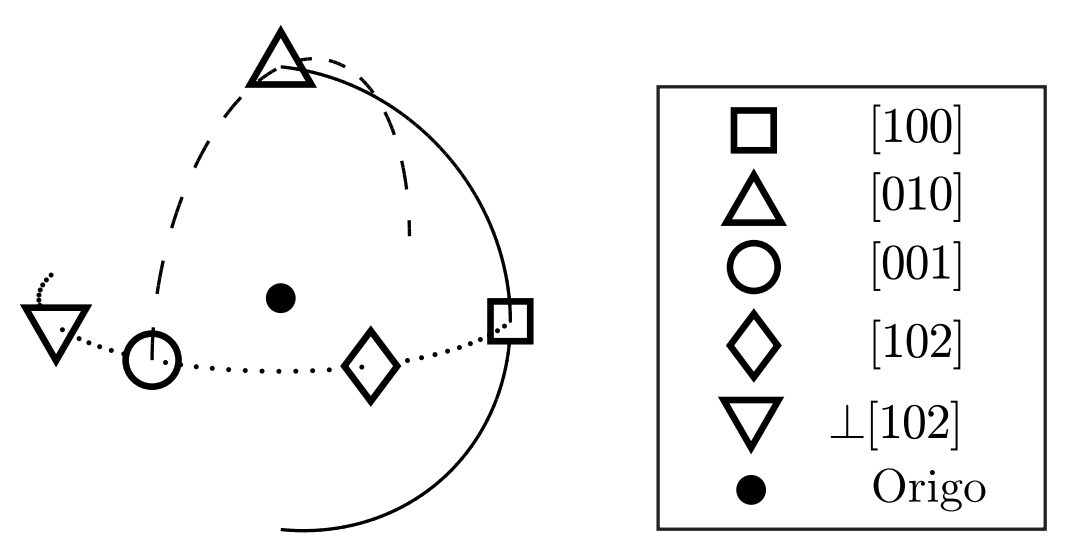




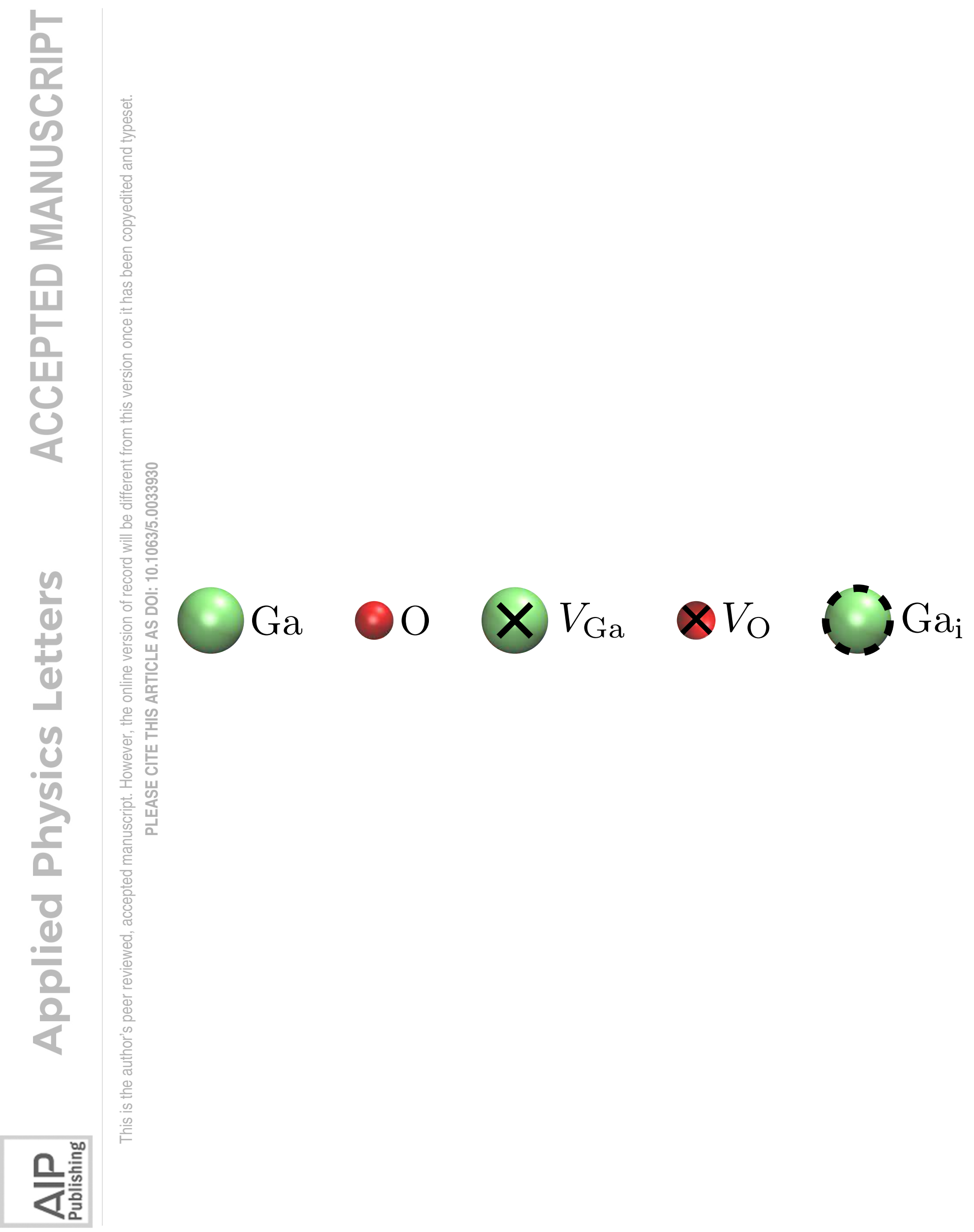




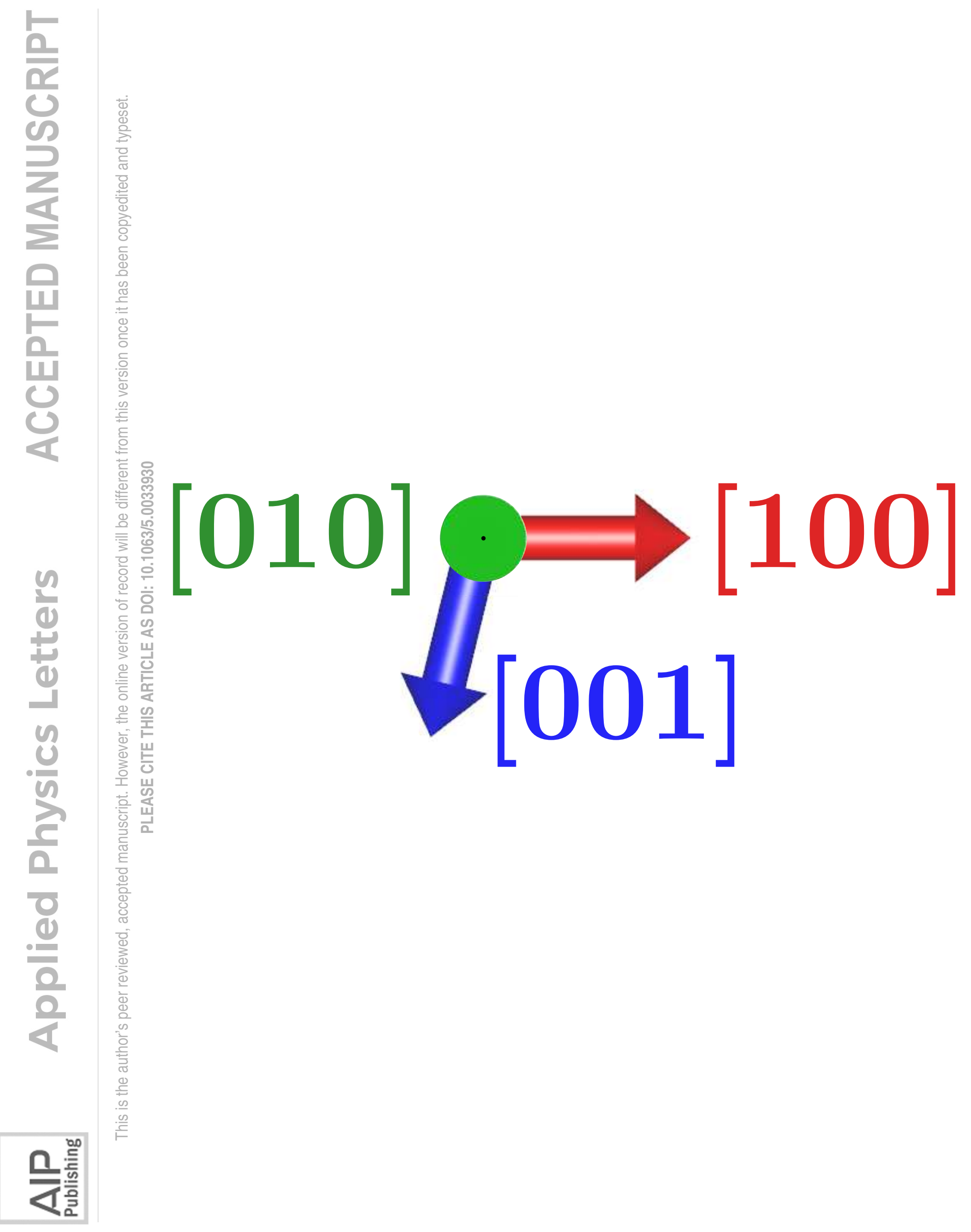




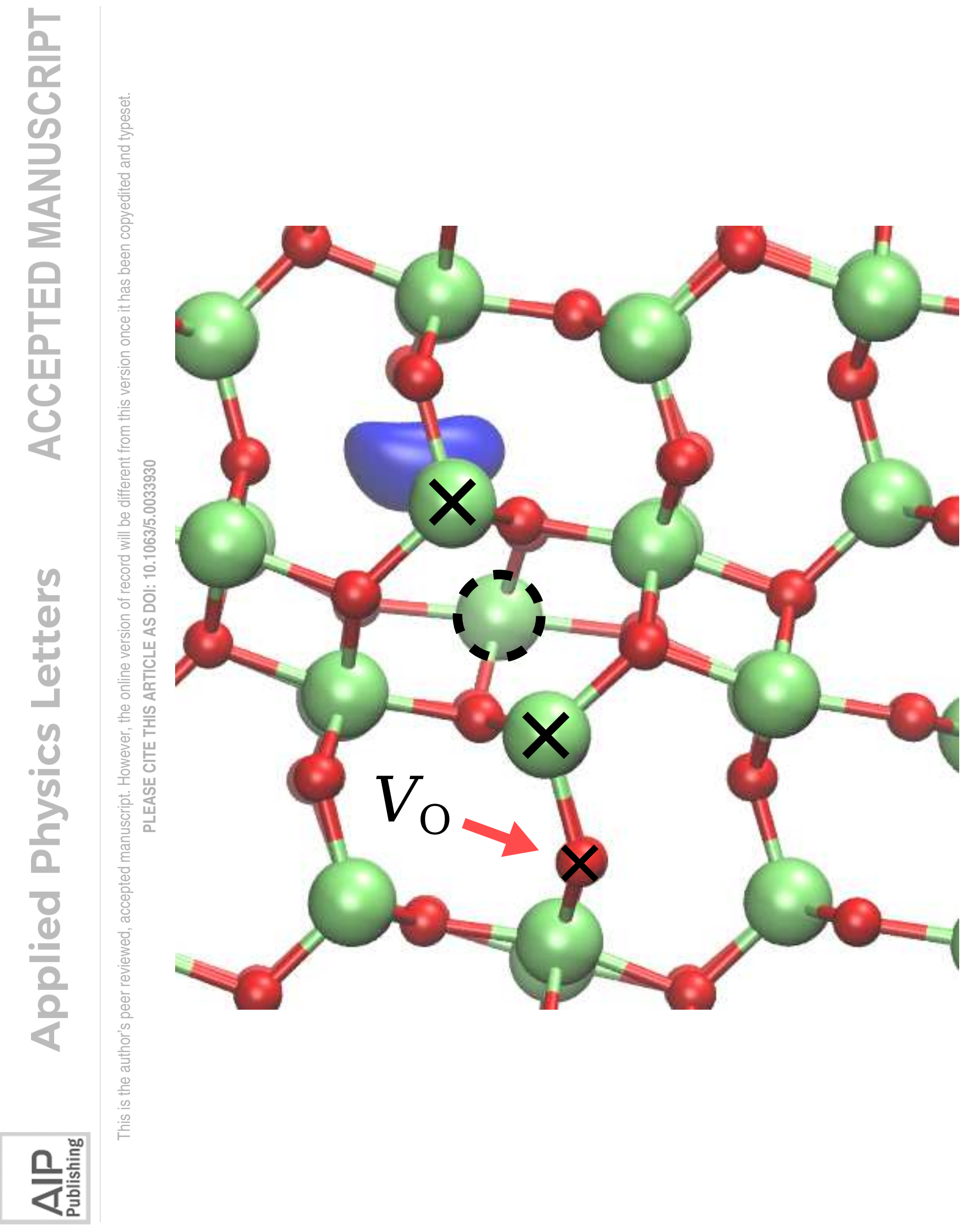




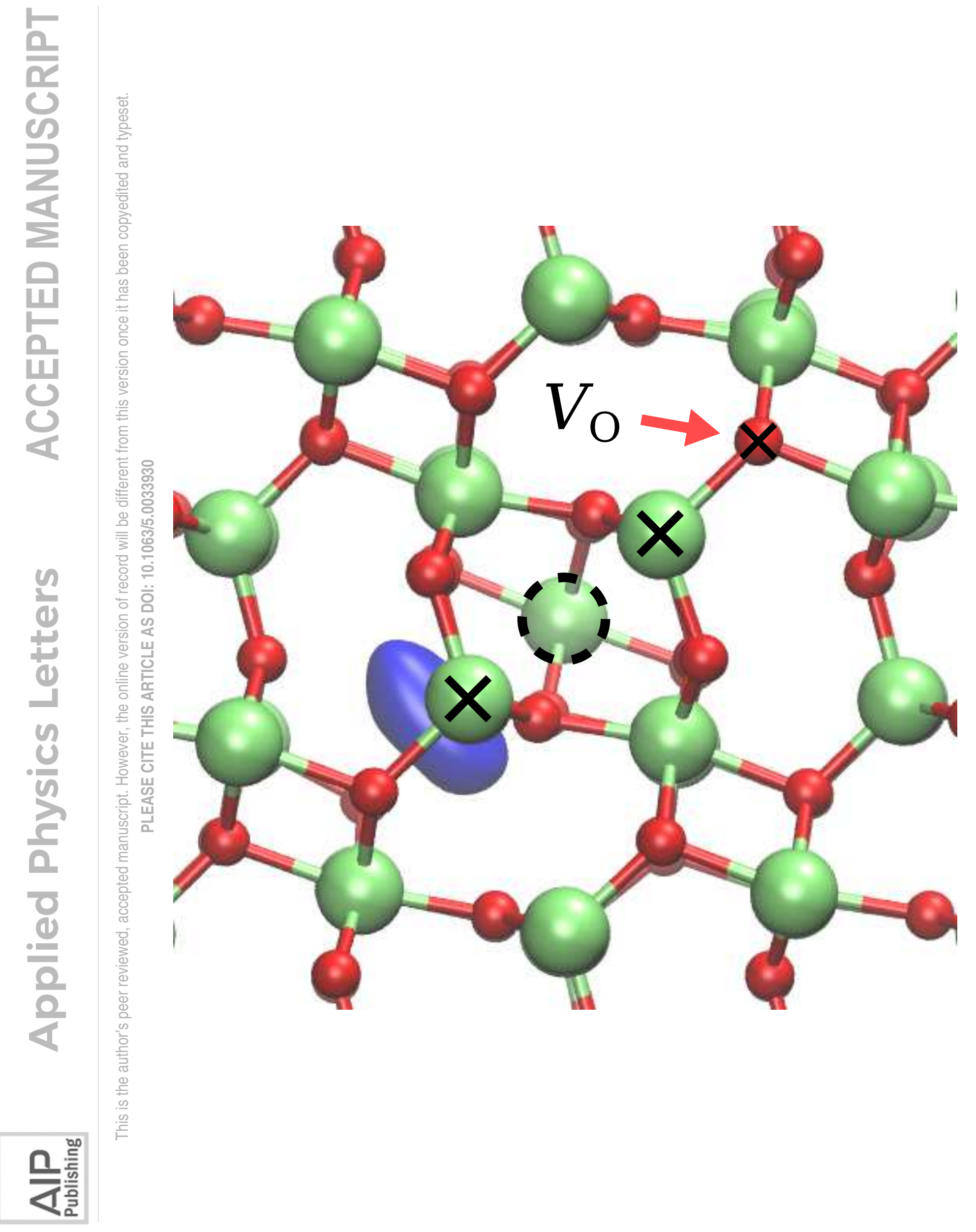




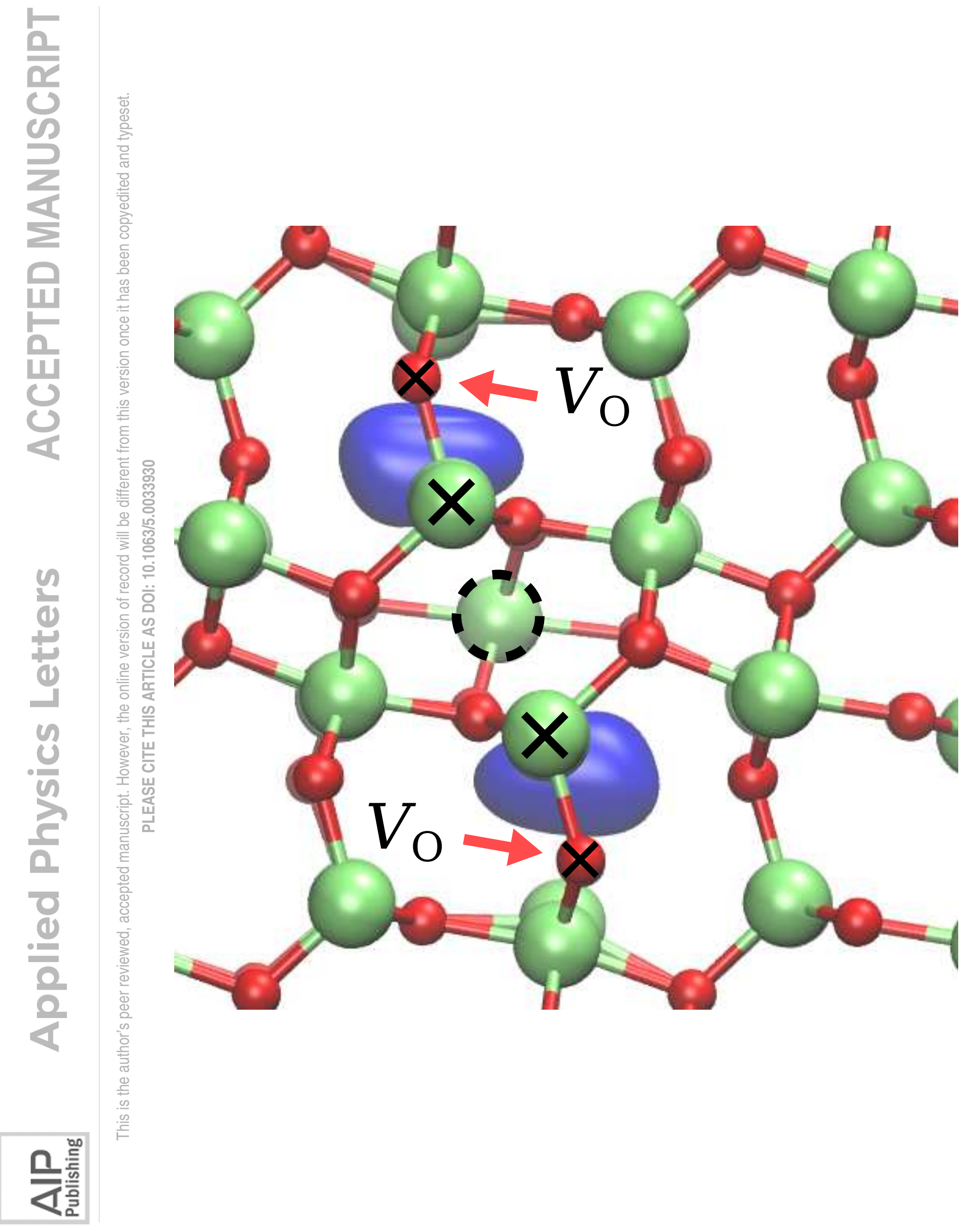




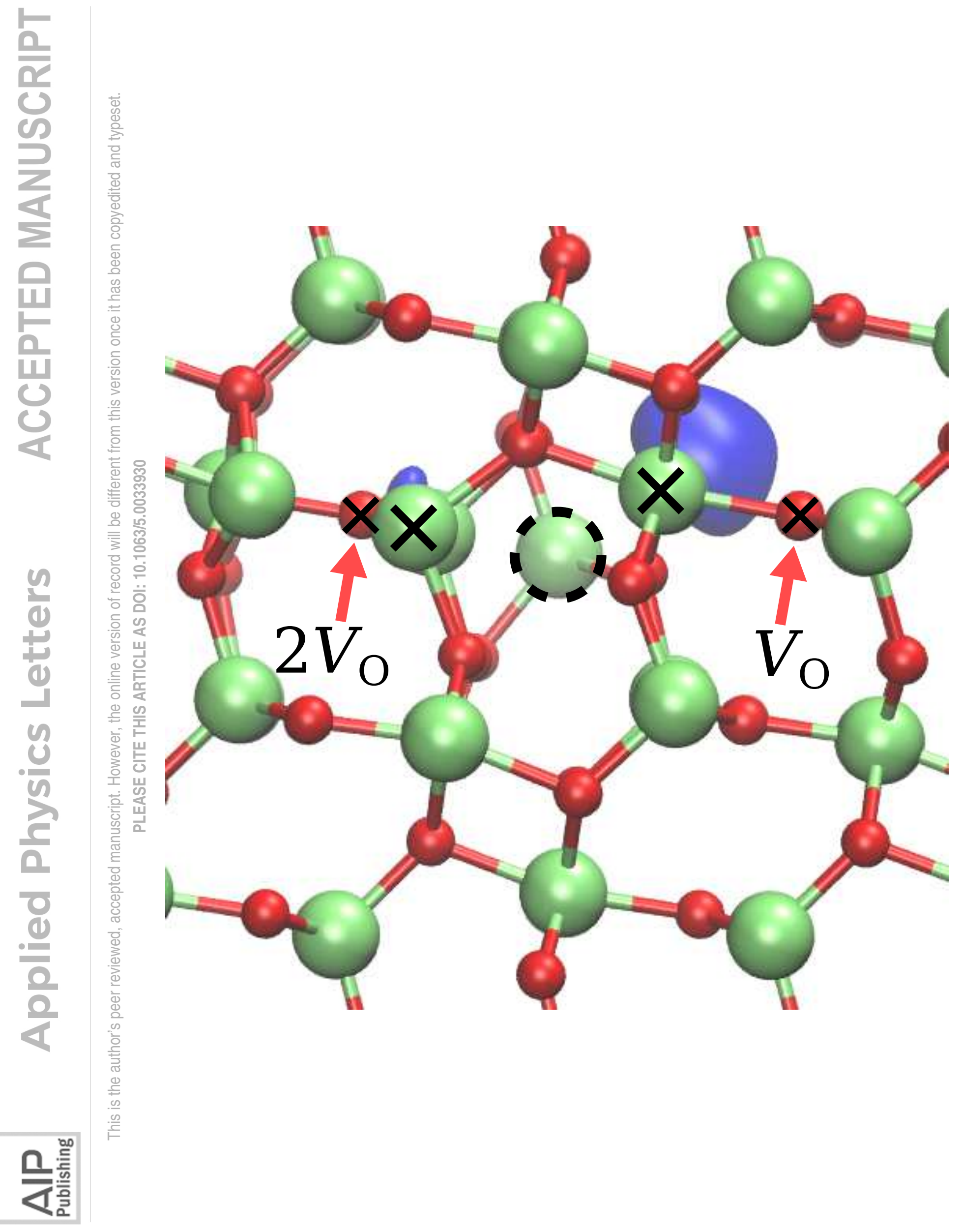




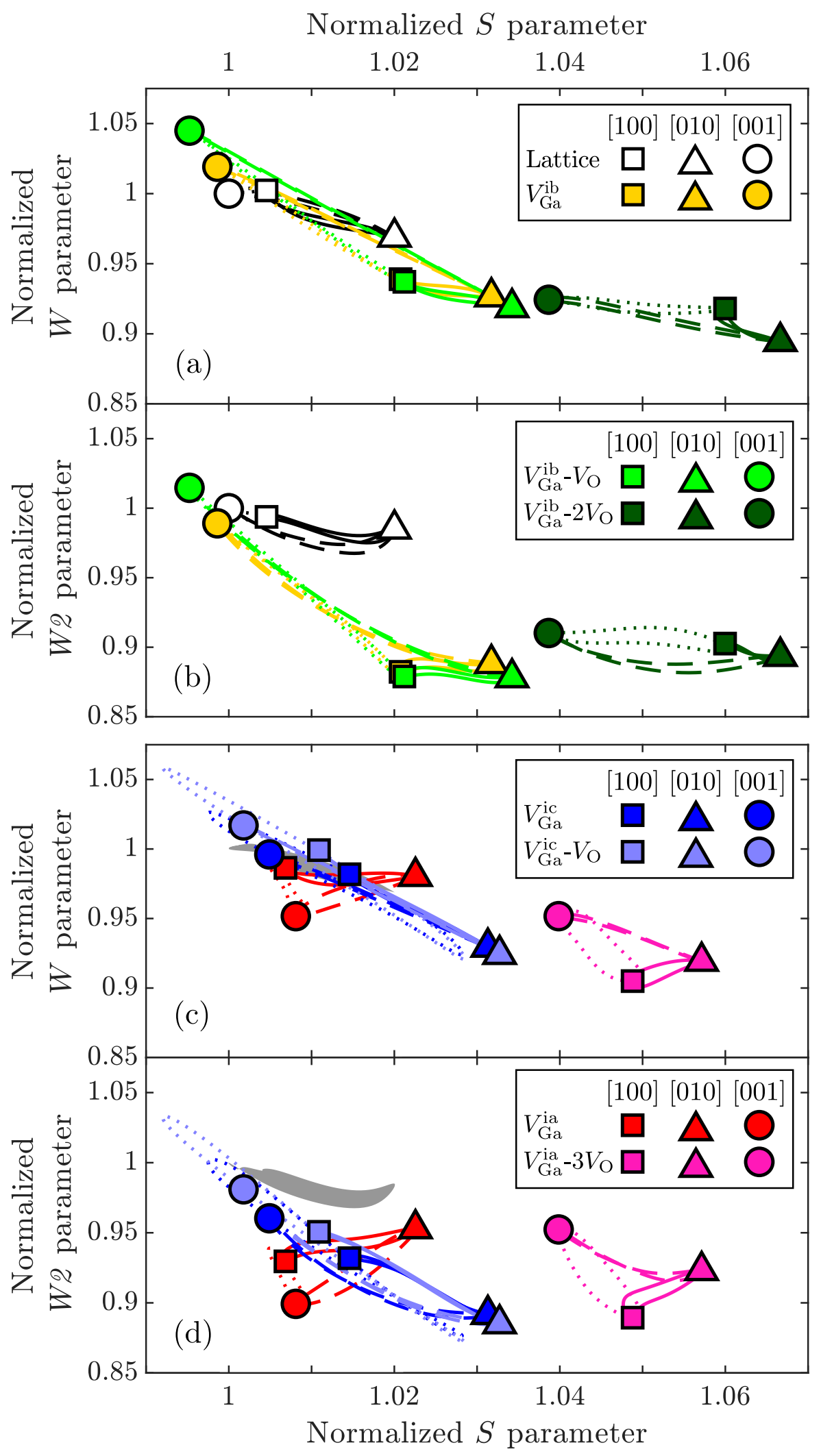




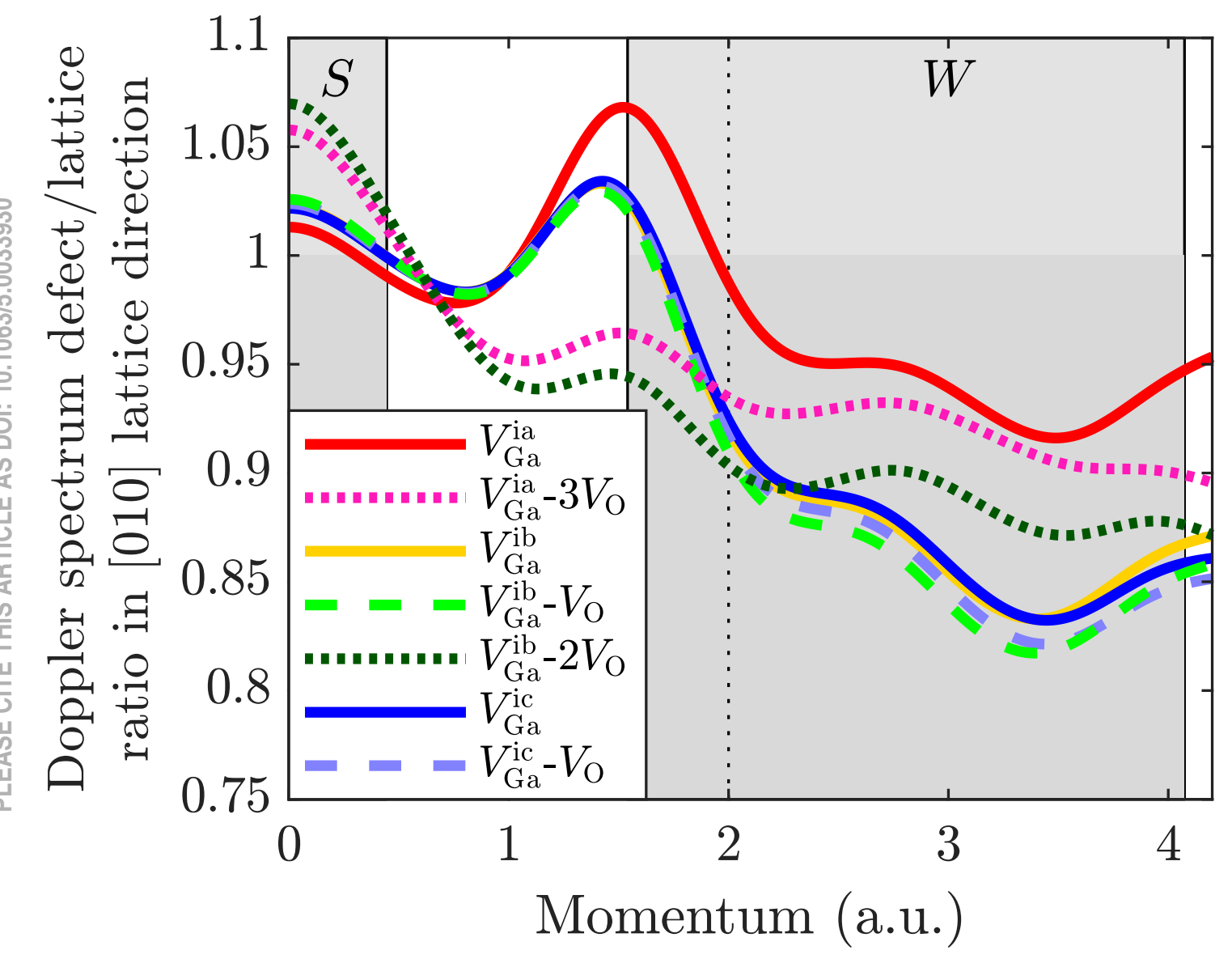

兄喜譬 